\title{
Personalized Web Search Architecture Supporting Privacy
}

\author{
Nidhi Saxena, Bineet Gupta
}

\begin{abstract}
Web is gigantic wellspring of information and data and each one relies upon it for accomplishing all kind of information. With the presence of enormous information and assortment of data it gets hard for end client to separate required data. Thus there comes the need to customize every client search so as to acquire important outcomes at the time of searching. This paper gives architecture of internet search utilizing an improved customized method which recognizes every client search keeping protection as a measure concern. The proposed plan is more valuable where more than one client get to single gadget for looking. A through depiction of architecture design gives total perspective on customized protection improved web search framework. The framework designed for varied interest holder clients demonstrates profoundly effective for every client individual need. The framework goes about as an improvement in the region of web mining as it expects to be easy to understand just as secure.
\end{abstract}

Keywords : Customization, Page ranking, Personalization, Privacy, Web search, Web Search log.

\section{INTRODUCTION}

Our reality spins around web and we are getting subject to World Wide Web for accomplishing any sort of information .Search engine is accessed by each period of individuals, for picking up assortment of data. Then again a large number of pages are included each day hence the size of data accessible on the web is expanding step by step. These outcomes in rising size of information on web. The developing size of information gives better help to client yet require extraordinary exertion while looking so as to get pertinent outcome. Sometimes user fails in getting required and intrigued data. One ordinary response for above issue is personalization, which tweaks the Web state of clients and causes them search their information need adequately by considering about their interest and requirements. Therefore search engine need to be upgraded all the time in order to satisfy user. Certain prevailing difficulties in web search incorporates-

- To decrease the quantity of wrong "hits"

- Interest based outcomes

- User Identification

- Security/Privacy

For customized results client is perceived by IP address and results are positioned dependent on search developed from framework.

Revised Manuscript Received on February 05, 2020

* Correspondence Author

Nidhi Saxena*, Computer Science, SRMGPC. Lucknow UP,

India.Email: nidhi.shivansh@gmail.com

Bineet Gupta, Computer Applications, SRMU, Lucknow UP, India.

Email:bkguptacs@gmail.com

(C) The Authors. Published by Blue Eyes Intelligence Engineering and Sciences Publication (BEIESP). This is an open access article under the CC BY-NC-ND license (http://creativecommons.org/licenses/by-nc-nd/4.0/)
Therefore it is a problem for providing search outcome as per user savor on alone system. The prime test lies in website page ranking is the means by which to consider each client as remarkable presence.

Our proposed engineering is an augmentation to personalization approach being utilized. It enable client to recognize his inquiry and accordingly gives results in user friendly approach. The framework gives inbuilt element of protection and security which is of significant concern with personalization.

\section{RELATED WORK}

The research in the field of web searching and personalization had been started in 90's .Lot of improvement have been made in this area. Each time improvements are made keeping in view different difficulties in web search. From few years searching inclined towards personalization .Improved techniques like Arbelaitziet al. in [1], shows the incessant course of action exhuming and bunching to adjusting the user's inclination and prerequisites. Pandey etl al. in [2], shows the work on large knowledge files. In this method personalized collaborative filtering is combined with association rule Mining and FP-growth algorithm. R. Thiyagarajan et al. in [3], exists his work for expectation of the users perusing action and afterward prescribes the website pages to the users as per the region of intrigue. Weighted k-means bunching calculation is applied here to foresee the users navigational conduct. Ahmad Hawalahiet al. in [4], proposed the methodology in worry of better provision of users conduct towards the site. Meera Narvekar et al. in [5], shows that World wide web such a significant number of sites are expanding because of that reason it is exceptionally hard to foresee or comprehend the users conduct towards the site. This methodology works in the forecast procedure. Ravi Khatri et al. in [6], proposed way to deal with give the pertinent material to the user in a specific time interim and furthermore gives improved customized web recommender model, which thinks about user explicit exercises and furthermore considers some other extra components identified with sites. Positioning website pages utilizing AI approaches is advanced by Sweahet al., [7].

Srouret al., [8] provided a personalized Web Page ranking using trust and resemblance. Shiguanget al., [9] given the improvement of page ranking algorithm based on time stamp and link. In [10] Yen et al. existed the changed increased information unearthing calculation for finding web traversal plans when the user links are embedded into and erased from 
unique knowledgebase. Kraft et. al [11] , Sugiyama et. al [12], Zhengyu Zhu et al [13], and Bounoy and Walairacht [14] have been contributed in enhancing the search results process by proposing techniques for users' preferences.

Zhou et al. [15] proposed high utility way traversal course of action exhuming, which brings the idea of utility into way traversal game plan uncovering model. An ongoing report by Whiteiet al. [16] is exploring the natural inclinations of search engines and their effect on the quality of material that contacts individuals.

\section{IDENTIFICATION OF PROBLEM}

There are many problems associated with user data collection. The usage data are usually obtained from either web server log, at the server side, or web browser, at the client side. Data acquisition via server log involves preprocessing which is an overhead. Unfortunately, cache hits are missing from the server log, rendering it as an incomplete source of information.

Temporal features such as page view-time of pages are considered highly informative in deducing user preferences However, timestamps recorded for each server log entry includes the network transfer time. Hence page-view time extracted from the server side is not accurate. User session identification and data cleansing are the most difficult and time-consuming tasks performed during preprocessing of the server log. Since there is a many-to-many relationship between users and IP addresses which are recorded in the log entry for each HTTP request, it becomes an unreliable source of information for user session identification. Hence server log is not reliable as a source of usage data for web usage findings.

HTTP request, it becomes an unreliable source of information for user session identification. Personalization uses user data whether taken explicitly or Implicitly. Since there is a many-to-many relationship between users and IP addresses which are recorded in the web log created on the server, outcome to overwhelming burden on server and furthermore prompts protection issues. A rundown of issues recognized in personalized internet searcher are recorded beneath -

- As the query history increments on server or PC computational multifaceted nature increments.

- Users face issues while determining the data they need

which results to wastage of time in searching by making useless hits.

- Some keyword used for search have more than one meaning and relevance then client need to give long query.

- If there are more than one user looking from same framework at that point results will be same for every client.

- Lack of security is by all accounts significant act of personalization.

So as to defeat the given issue there is a need of a personalized architectural framework which can give pertinent list of ranked pages in productive manner.

\section{PROPOSED PERSONALIZED WEB SEARCH ARCHITECTURE}

To avoid all these problems the architecture proposed in this paper suggests a client-side web usage data acquisition. The proposed personalized search system works by extracting search results from the existing search engine and re-ranking the results based on personalization.

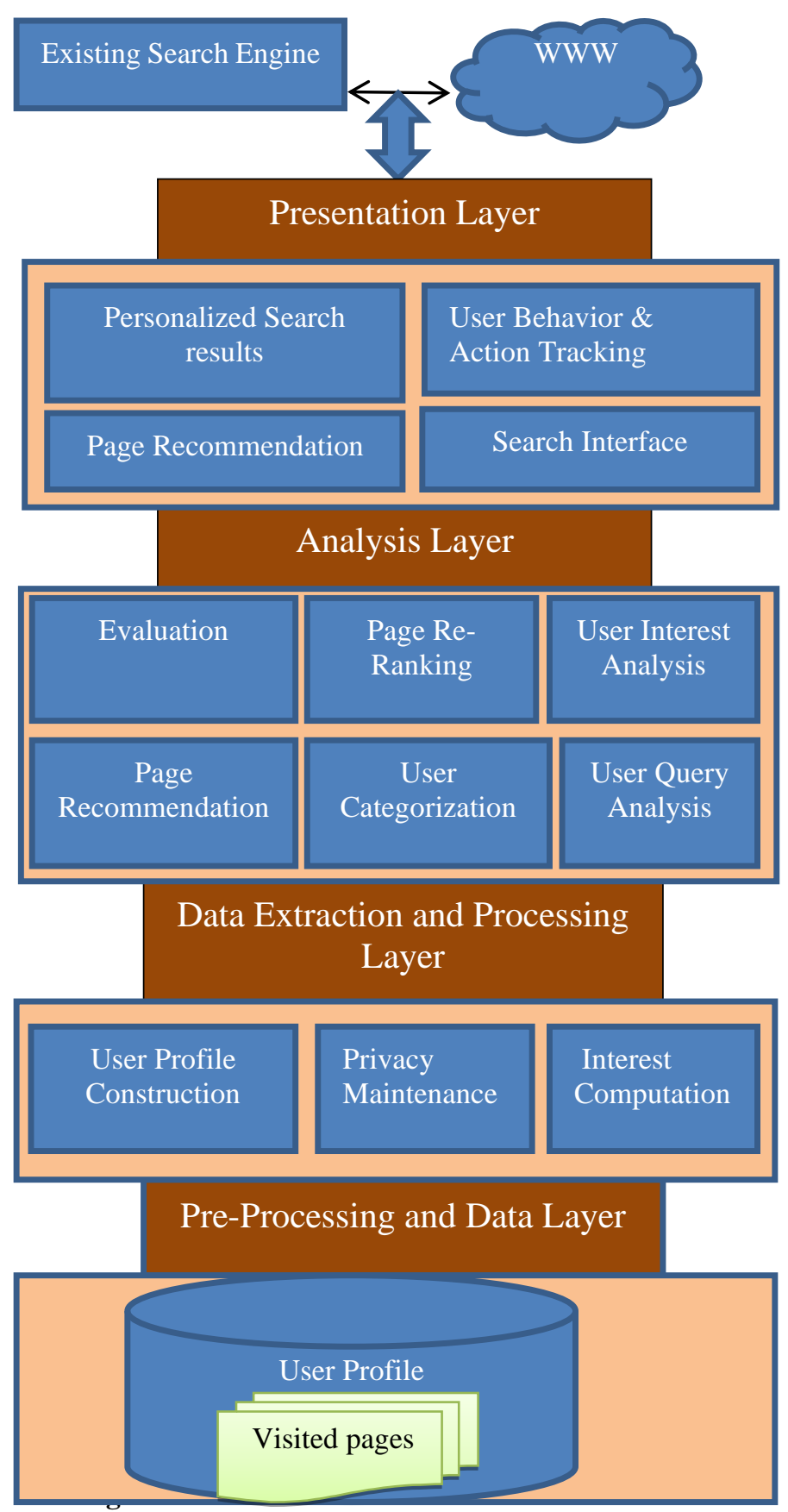

The architecture is made up of the following six layers: Presentation, Analysis Layer, Data Extraction and Processing layer, Data layer.

Each layer in the architecture performs unique functionality and together facilitates the process of providing relevant result to number of users on single machine. 


\section{A. Presentation Layer}

The presentation layer is the place where the user interacts with the personalized search system and the data about the user's search is collected.

Presentation layer perform following tasks-

- Search interface

- Personalize search results

- User behavior and action tracking

- Page recommendation

The presentation layer keeps track of user preferences while searching from the machines, from where the user performs the search. Users submit their search queries through a specially designed browser and view the result pages. The user queries are submitted to an existing search engine like Google or Yahoo!. Presentation layer collects top results given out by the existing search engine. These data are analyzed, re-ranked based on page relevancy with respect to the user interests and current context of search. The pages are then recommended to the users through the browser. The user interface is designed in such a way that it keeps track of user data like the search queries given, pages visited, time spent on a page.

The tracked user information is deposited into the data layer after pre-processing. Thus the user data is collected implicitly from the users without any user intervention.

Table I: List of User's Behaviors (Implicit Indicators) Gathered by the Browser User Behavior Remarks

\begin{tabular}{|l|l|}
\hline User Behavior & Remarks \\
\hline Anonymous User Name & $\begin{array}{l}\text { Essential for any user to use the } \\
\text { browser \& to perform search } \\
\text { through the WWW }\end{array}$ \\
\hline Information Viewed & $\begin{array}{l}\text { Textual Contents of a visited } \\
\text { page that is viewed }\end{array}$ \\
\hline URL of the accessed page & $\begin{array}{l}\text { Collected and displayed in } \\
\text { graphs }\end{array}$ \\
\hline Page-view time & $\begin{array}{l}\text { Measured in seconds normalized } \\
\text { by page size }\end{array}$ \\
\hline Search Queries & $\begin{array}{l}\text { Essential for any search \& direct } \\
\text { indicators of user's information } \\
\text { need. Utilized for user and page } \\
\text { categorization. }\end{array}$ \\
\hline
\end{tabular}

Privacy policy statements are often confusing and arouse suspicion in users' minds, leading to reluctance to share personal information. To overcome this, search log is maintained on client and user login into the search engine through face recognition method which separates each user search on a single machine. The users of personalized search system are with anonymous user identity acting as username and password. Hence the problem of privacy is also eliminated. The user data thus remains on client with distinct web log.

\section{B. Pre- Processing and Data Layer}

The user data tracked by the browser is raw data like the search query string, URLs of web pages, time in seconds, etc. Hence pre-processing is done to extract important concepts to represent the content of such unstructured/semi-structured documents. The visited pages are maintained by indexing their URLs with the concepts extracted from the pages. Such keywords that are used for indexing the URLs are called as Index Word (IW).
The data collected by the browser from the user end are populated into tables present in the data layer. The data layer acts as a central warehouse of all the data needed for achieving an effective personalization. Every web page visited by the user is represented by the page's URL. The user data is organized in special structures called the transactions and search sessions.

The data layer is the source for data extraction, knowledge and analysis. The data storage layer houses the initial user profiles which are constructed automatically. User's interests are identified automatically from the collection of various search queries and the relevant pages visited by the user. The terms in the user's search queries, the Index Word (IW) of the visited pages, the frequency of usage of similar search terms indirectly highlights the user's interest in a particular concept.

The search interface works by recognizing user as new user or old user. The proposed re-ranking procedure works for old user thereby personalizing the result. Beside user searched data the web log maintained on the client machine stores URL searched by all users for each query. This data increases as the number of user increases or search query increases.

\section{Data Extraction and Processing Layer}

The data extraction is essential for deriving the conceptual relations between the search queries and their relevant pages, structuring the personalized information needed for final re-ranking process.

The data extraction layer also structures the users search behavior as a graph, identifies relevant and irrelevant pages and hence this layer aids filtering of irrelevant pages according to the current context of search. User profiles store the approximation of user's interests and disinterests.

In this work the methodology used for the generation of user profiles differs from the majority of other approaches. The profiles are: generated automatically, without explicit user feedback, and dynamic, i.e., not based on a fixed period of time, but it evolves over time.

In a nutshell, the Data extraction and processing layer does the following functions:

- Relevance Computation based on page-hit, page-view time, query hit.

- Construction of Privacy Controlled Personalization using face recognition and separate search log on client .

- Automatic construction of user profiles called Personalized Page-View.

This layer extracts the spatial data like the pages visited and their hits, search queries used and their hits, the temporal data like page-view time and the implicit indicators of user interests like user actions on pages. Such information is used to compute the Personalization.

\section{Analysis Layer}

Analysis of user behaviors, interests, search paths are thus very essential for final page re-ranking. Also this layer utilizes user search log for labeling user interests and categorizing such interest labels into long and short- term interests. The data analysis layer plays a vital role. It perform following tasks- 
- User Interest analysis

- Evaluation of relevant pages

- Page re-ranking based on each user interest

- User search query analysis for old or new query

- User categorization as old user or new user

- Page recommendation based on user interest

The analysis layer works at each phase of personalization. It starts with the analysis of user interest based on track record. User interest is evaluated by analyzing searched Urls, frequency of visited page and the total time spend on each page. The time spend also depends on size of web document. The analysis acts as a basis of personalized search.

User interest analysis helps in the evaluation of relevant page by applying mathematical formula for finding weight of each web page. The pages returned by base search engine are re-ranked based on user interest.

. The proposed system performs analysis for distinguishing new user and old user. The user is identified by face matching method. The personalization method works only in the case of old user where the track record and personal detail are available on user log. On the basis of analysis, web pages are recommended to the user. In a multi user search environment search paths of individual users are identified. Search behaviors identification act as a source of page recommendations for each user. Analysis of user behaviors, interests, search paths are thus very essential for final page re-ranking.

The results of all the above mentioned analysis are combined and utilized for final page re-ranking and recommendation. The various analyses that are performed for page re-ranking in the analysis layer are based on each user separately which provide personalized interface for each user on same interface. Finally the re-ranked pages are displayed to the user

\section{PERSONALIZATION COMPUTATION}

A customized re-positioning system is applied to give clients increasingly important searched results top positioned. Web Search personalization is accomplished in 3 stages:

- Recognizing client's point of enthusiasm of current search.

- Calculate weight of web pages based on parameter applied.

- Re-rank the web pages based on relevancy weights.

As an initial step, the question put together by the user is coordinated to the client profile to pick the previous viewed and saw pages for indistinguishable search. If the identical match found then next step will be proceeded otherwise search results from existing search engine without personalization becomes the final result. The returned web pages get stored in the web log.

After selecting the web pages based on user importance the weight of each page is calculated for ranking. The web page will be chronologically displayed from highest to lowest weight. For calculating the weight of each web page subsequent parameters are measured-

- Inspected page Frequency

- Time spend on each selected page.

\section{A. Page Frequency}

Frequency characterizes the occasions the page is assessed by client in the prior search. The Inspecting Frequency of a page $u$ is the occasions that a page examined after page v. As the frequency of a web page inspected increases its probability of top rank also increases. It is measured by increasing the count by 1 each time the page is inspected by the same user.

The frequency weight (FW) is defined here-

$$
F W(u) \frac{\text { Number of Vist on a page }(u)}{\text { Total number of visit onall pages }} \times P R(u)
$$

\section{B. Time Spend}

Time spent on a page recreates the overall result of each page, in light of the fact that a user for the most part invest more energy in a needful page and rapidly jump to other page. The formula to calculate the weight based on time spent on each page is-

$T W(u)=\frac{\text { Time spend on a page }(u) / \text { page size }(u)}{\operatorname{Max}_{u \in U}(\text { Time spend on a page }(u) / \text { Page size }(u))}$

Frequency along with time spend provides proper interest of user in a web page. Sometimes user inspects a page but do not show much interest in that page and close it therefore if user selects a page that does not reflect that user is interested in that page. Therefore if user opens a web page and spend sometimes on it then we can consider page relevancy.

$W(u)=F W(u)+T W(u)$

\section{PRIVACY PRESEVATION}

Other than personalization the designed Architecture intends to give privacy upgraded customized search. The system gives privacy in the accompanying way-

- The proposed system works on client account and Framework works on client machine, consequently all data are not disclosed on web.

- User face is matched before searching for which limits unapproved get to.

- Each user has separate profile and can't view other client detail.

Here a user side personalization is proposed which manages the saving security and privacy to completely ensure client protection.

For protection and personalization two fundamental strategies were advanced: Automatic making of client profiles dependent on our profile generator component and then again suggest framework dependent on the substance to gauges the client interest dependent on user side meta data 
Client side profile

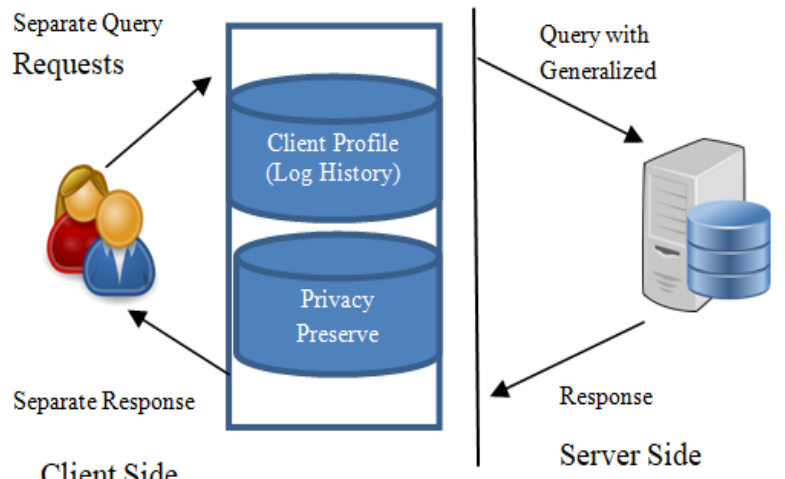

Networking

Fig. 2. Privacy Preserving Personalization Model

Fig. 2. shows how privacy is preserved while personalization. The proposed engineering works as a customer side instrument and here we shield the information from the server, so just we give a security to the client. Each query from the client were given by the different request to the server. The server response using base search engine. These results are re-ranked on client side using user personal profile therefore the system shields all details from server. From that point onward, online profiler question were prepared in the way of speculation process, it is applied to meet the particular essentials to deal with the client profile and it depends on the preprocessing the client profiles. The delicate relevant material is normally not a fundamental angle since it is carefully put away and applied on the user side. A client individual material including client queries and click logs history lives on the clients PC. Here it uses the acquired strategy to generalize the query. It permits playing out the customization procedure to secure the information and apply the User adaptable Privacy-safeguarding Search system tended to the protection issues. This point is to customize results at the same time securing the protection in singular user profiles.

\section{RESULT ANALYSIS}

The enactment of the anticipated procedure can be estimated in terms of precision, recall and f-measure. Precision, Recall and F-measure are determined as beneath -

\section{A. Precision}

Precision is the part of archives recovered that are applicable to a client material needs. It considers all recovered data.

\section{Precision $=\frac{\text { Number of Relevant retrieved documents }}{\text { Total number of retrieved documents }}$}

\section{B. Recall}

Recall is the division of archives effectively recovered and pertinent to an inquiry. Also termed as sensitivity, it can be looked at as the prospect that a significant document is recovered by the query.

\section{Recall $=\frac{\text { Number of Relevant retrieved documents }}{\text { Total number of documents in the collection }}$}

\section{F-measure}

F-measure is the mean of Precision and recall, and provides abundant results when precision and recall give good consequences.

\section{$\mathrm{F}-$ measure $=2 \cdot \frac{\text { Precision } 8 \text { Recall }}{\text { Precision }+ \text { Recall }}$}

(6)

To test the performance of the proposed work Precision Recall and F-measure is calculated. For the purpose of experiment data set for three users User1 , User2 and User 3 is taken. Query "kingfisher" is used for experiment.

Table II. User1 Track record for query "kingfisher"

\begin{tabular}{|l|c|c|l|l|}
\hline Pages & Page rank & $\begin{array}{l}\text { No. of } \\
\text { inspects } \\
\text { on web } \\
\text { page(u) }\end{array}$ & $\begin{array}{l}\text { Time } \\
\text { spend on a } \\
\text { Page (u) }\end{array}$ & $\begin{array}{l}\text { Page } \\
\text { size(u) in } \\
\text { pages }\end{array}$ \\
\hline AR(bird) & 0.18 & 1 & .30 min, & 4pages \\
\hline B(Beer) & 0.1 & 1 & .20 min & 3 pages \\
\hline C(Airlines) & 0.17 & 3 & $\begin{array}{l}2 \mathrm{~min}, 1.5 \\
\text { min, } 1 \text { min }\end{array}$ & 2 pages \\
\hline D(bird) & 0.12 & 0 & & 2 pages \\
\hline E(Airlines) & 0.09 & 4 & $\begin{array}{l}1.5 \text { min, } 1 \\
\text { min, } 2 \text { min, } \\
2.5 \text { min }\end{array}$ & 4 pages \\
\hline F(Airlines) & 0.06 & 3 & $\begin{array}{l}1 \mathrm{~min}, 2 \\
\text { min, } 1.5\end{array}$ & 3 pages \\
\hline
\end{tabular}

Table III. User2 Track record for query "kingfisher"

\begin{tabular}{|l|c|c|c|c|}
\hline Pages & Page rank & $\begin{array}{l}\text { No. of } \\
\text { inspects } \\
\text { on web } \\
\text { page(u) }\end{array}$ & $\begin{array}{l}\text { Time } \\
\text { spend on a } \\
\text { Page (u) }\end{array}$ & $\begin{array}{l}\text { Page } \\
\text { size(u) in } \\
\text { pages }\end{array}$ \\
\hline PR(u) & 0.18 & 3 & $\begin{array}{c}1 \mathrm{~min}, \\
2 \mathrm{~min}, 1.5\end{array}$ & 4pages \\
\hline B(Beer) & 0.1 & 1 & $.20 \mathrm{~min}$ & 3 pages \\
\hline C(Airlines) & 0.17 & 1 & $.40 \mathrm{~min}$ & 2 pages \\
\hline D(bird) & 0.12 & 3 & $\begin{array}{l}1 \text { min, } 2 \\
\text { min, } 2.5\end{array}$ & 2 pages \\
\hline E(Airlines) & 0.09 & 0 & & 4 pages \\
\hline F(Airlines) & 0.06 & 1 & $1 \mathrm{~min}$ & 3 pages \\
\hline
\end{tabular}

Table IV. User3 Track record for query "kingfisher"

\begin{tabular}{|l|c|c|c|c|}
\hline Pages & Page rank & $\begin{array}{l}\text { No. of } \\
\text { inspects } \\
\text { on web } \\
\text { page(u) } \\
\text { (static) }\end{array}$ & $\begin{array}{l}\text { Time } \\
\text { spend on a } \\
\text { Page (u) }\end{array}$ & $\begin{array}{l}\text { Page } \\
\text { size(u) in } \\
\text { pages }\end{array}$ \\
\hline A(bird) & 0.18 & 1 & .20 min & 4pages \\
\hline B(Beer) & 0.1 & 3 & $\begin{array}{c}1 \text { min, } \\
2 \text { min,1.5 }\end{array}$ & 3 pages \\
\hline C(Airlines) & 0.17 & 1 & .40 min & 2 pages \\
\hline D(bird) & 0.12 & 0 & & 2 pages \\
\hline E(Airlines) & 0.09 & 0 & & 4 pages \\
\hline
\end{tabular}


From the data set the user behavior and interest can be judged. User 1 is interested in Airlines kingfisher therefore the web documents displayed having related results are relevant while others are irrelevant. User 2 is interested in Bird named kingfisher and User3 is interested in beer named kingfisher. The interest of all three varies but the existing personalization search system provides ranking of web pages based on IP and not on individual user. Therefore results may not be relevant to others. The relevancy calculation technique using Precision, Recall and F-measure is applied in the earlier given example to verify the accuracy of proposed system as compared to earlier approach. Based on user interest the displayed pages are marked as "hit" or "miss" . A web page is said to be a "Hit" if it is of some interest to user and "miss" if it is irrelevant to user.

Consider the data set of Table II, III and IV where six web pages- A,B,C,D,E,F are taken for assessment . Out of them Hits and miss for User1, User2 and User3 are shown in the Table V.

Table V: Web page relevancy for each user

\begin{tabular}{|l|l|l|l|}
\hline Web pages & User1 & User2 & User3 \\
\hline A & Miss & Hit & Miss \\
\hline B & Miss & Miss & Hit \\
\hline C & Hit & Miss & Miss \\
\hline D & Miss & Hit & Miss \\
\hline E & Hit & Miss & Miss \\
\hline F & Hit & Miss & Miss \\
\hline
\end{tabular}

Table VI: Performance evaluation of Existing search engine without User Identification

\begin{tabular}{|l|l|l|l|}
\hline User & Precision & Recall & F-measure \\
\hline User1 & 0.3 & 1 & 0.46 \\
\hline User2 & 1 & 1 & 1 \\
\hline User3 & 0.6 & 1 & 0.75 \\
\hline
\end{tabular}

Table VII: Performance evaluation for Proposed Architecture (User Identification Based Personalization)

\begin{tabular}{|l|l|l|l|}
\hline User & Precision & Recall & F-measure \\
\hline User1 & 1 & 1 & 1 \\
\hline User2 & 1 & 1 & 1 \\
\hline User3 & 1 & 1 & 1 \\
\hline
\end{tabular}

In the Table $\mathrm{V}$ the precision and $\mathrm{F}$-measure is low for user 1 and user 3 while considering top three search results without user identification. It does not provide relevant results to all users as compared to Personalization based on user Identification which provides relevant results to all users depicted in table VI

The comparison between two results can better be analyzed with the help of bar chart shown in the Figure followed.

\section{Performance Evaluation}

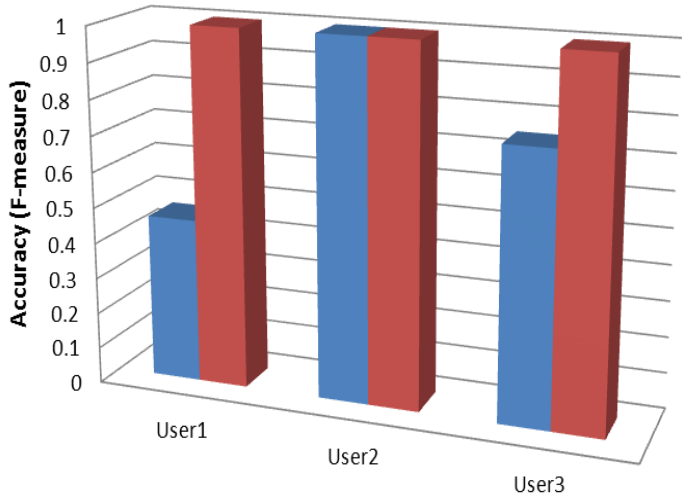

\begin{tabular}{|c|c|c|c|}
\cline { 2 - 4 } \multicolumn{1}{c|}{} & User1 & User2 & User3 \\
\hline $\begin{array}{c}\text { Existing Personalization } \\
\text { Search system }\end{array}$ & 0.46 & 1 & 0.75 \\
\hline $\begin{array}{c}\text { Proposed Personalization } \\
\text { System }\end{array}$ & 1 & 1 & 1 \\
\hline
\end{tabular}

Fig. 3. Relevancy Evaluation using F-measure

The performance of the anticipated system is depicted in the given bar chart. The comparative study of existing personalization system with proposed system is measured in terms of Precision, Recall and F-measure. It can be seen that this novel designed architecture is providing relevant results to all three user accessing same system for searching even if their interest vary. While other algorithm works on IP therefore calculate single value for Precision, Recall and F-measure which does not provide relevant result to all user. In the Table VI User 2 is getting relevant result in earlier approach andUser1 and User3 are getting irrelevant results which reduces F-measure. But in proposed designed, users are identified and algorithm work separately on each user search log, the F- measure increases and Relevancy of result increases depicted in Table VII

\section{CONCLUSIONS}

Architecture of the proposed system provides the total philosophy for Personalized Search environment, which is a novel expansion in the field of filtering and ranking. This examination approach applied to the search engine will diminishes the dimensionality of the issue, aligned with ranking. This exploration brings about compelling web personalization and search by giving an underlying proportion of the necessities and interests of the client networks.

The proposed system handles the problem of illegal transfer of user personal track record from search history and sustains a secured personalized system. The privacy system is doubled with the application of Face recognition before searching. The result analysis performed ensures that the system provides effective and relevant results to all users 
There is a necessity to adopt such features while maintaining search log in home or in office where more than one user is using the same system for search. The system can act as a basis for higher scale search engine.

\section{REFERENCES}

1. E. Pariser :The Filter Bubble: What The Internet Is Hiding From You. Penguin Press, London,UK, 2011.

2. L. Parramore :The Filter Bubble. The Atlantic, October http://www.theatlantic.com/daily-dish/archive/2010/10/the-filter-bub ble/181427/. 2010.

3. G. Pandey, S. Patel, V. Singhal, A. Kansara,"A Process Oriented Perception of Personalization Techniques in Web Mining", International Journal of Science and Modern Engineering (IJISME),vol. 1, no.2, pp. 26-30, 2013.

4. R. Thiyagarajan, K. Thangavel, R. Rathipriya," Recommendation of Web Pages using Weighted K-Means Clustering", International Journal of Computer Applications , vol. 86 no. 14, pp. 44-48, 2014.

5. A.Hawalah and M. Fasli, "Dynamic user profiles for web personalization," Journal of Expert Systems with Applications , vol.42 no. 5,ACM,2015.

6. M. Narvekar, S. S. Banu," Predicting Users Web Navigation Behavior Using Hybrid Approach", Proceedings of International Conference on Advanced Computing Technologies and Applications (ICACTA-2015), 2015, pp. 3-12.

7. B. J. Jansen, A. Spink and T. Saracevic, "Real life, real users, an real needs: A study and analysis of user queries on the web", Information Processing \& Management, vol. 36, no. 2, pp. 207-227, 2000.

8. P. Lopez, B. Roy," Dyanamic Recomendation System Using Web Usage Mining For E-Commerce Users," Proceesings Computer Science 45-2015, pp. 60- 69, 2015.

9. J. Purra. Swedes Online: You Are More Tracked Than You Think. 2015.

10. T. Jing, W. L. Zou, and B. Z. Zhang, “ An Efficient Web Traversal Pattern Mining algorithm Based On Suffix Array", Proceedings of the 3rd International Conference on Machine Learning and Cybernetics,, 2004, pp.1535-1539.

11. B. J. Jansen, A. Spink and T. Saracevic, "Real life, real users, and real needs: A study and analysis of user queries on the web", Information Processing \& Management, vol. 36, no. 2, pp. 207-227, 2000.

12. U. Manber, A. Patel, and J. Robison, "Experience with personalization on Yahoo!," Comm. of the ACM, vol. 43, no. 8, pp. 35-39, 2000.

13. C. M. Karat, C. Brodie, J. Karat, J. Vergo, and S. R. Alpert, "Personalizing the user experience on ibm.com," IBM Systems Journal, vol. 42, no. 4, pp. 686-701, 2003.

14. B. Ives and G. Piccoli : Custom made apparel and individualized service at Lands' End, Comm. Of the AIS, vol. 11, no. 1, pp. 11-26, 2003.

15. F. Roesner, T. Kohno and D. Wetherall: Detecting And Defending Against Third-partyTracking On The Web, In Symposium on Networked System Design and Implementation, SanJose, California, USA, April 2012.

16. G. Soeller, K. Karahalios, C. Sandvig, and C. Wilson, "Mapwatch Detecting And MonitoringInternational Border Personalization," Proceedings of Online Maps International World Wide Web Conference, Montr eal, Canada, April 2016.

17. J. Huang and B. Gu, "Marketing Issues of Web Personalization: a Systematic Review, Classification and Conceptual Framework," journal of Residuals Science \& Technology, vol. 13, no. 8, 2016.

18. J. Van Doorn, and J.C. Hoekstra, "Customization of online advertising: The role of intrusiveness," Marketing Letters, vol.24,pp. 339-351, 2013.

19. D. Parra and P. Brusilovsky, "User-controllable personalization: A case study with Set Fusion," International Journal of Human-Computer Studies, vol. 78, pp. 43-67, 2015.

\section{AUTHORS PROFILE}

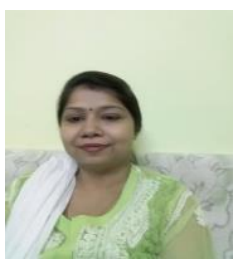

Nidhi Saxena is working as Assistant Professor in Computer Science department, Shri Ramswaroop Group of Professional Colleges, Lucknow UP, India from more than 10 years. She is a research scholar in Computer Science from Shri Ramswaroop Memorial University Lucknow Deva Road UP, India and submitted her Ph. D. thesis in web mining. Her area of research are Data mining, Web mining, Information retrieval and Security. She has published research papers in various reputed journals and international conferences.

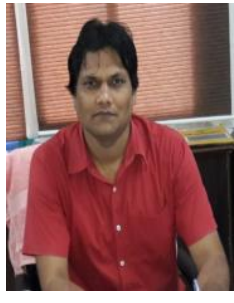

Dr. Bineet Kumar Gupta is working as an Associate Professor and Head, Department of Computer Application, Institute of Technology, Shri Ramswaroop Memorial University Lucknow Deva Road UP, India. Dr. Gupta has about 10 years of Higher education teaching and research experience at National and International platforms. His area of research are E- learning, Information Retrieval and Security, Software Engineering, Human Computer Interaction, ICT etc. He has published more than 30 research papers in various journals, conferences, and symposium of repute. 